\title{
Accurate object localization in gray level images using the center of gravity measure; accuracy versus precision
}

\author{
H.C van Assen $^{\dagger 1}$, M. Egmont-Petersen ${ }^{\dagger 2}$, J.H.C. Reiber ${ }^{\dagger 3} *$
}

\begin{abstract}
Computing the accurate localization of objects often requires a two-step approach. First, the coarse object detection is performed followed by accurate object localization. A widely used estimate of the object center with sub-pixel precision is the weighted center of gravity (COG). We derive a maximum-likelihood estimator for the variance of the (2D and 3D) COG, and an approximation to the estimate, as a function of the noise in the image. We assume that the noise in the image is additive, Gaussian distributed and independent between neighboring pixels.

Experiments using 2,500 generated markers with a cosine profile and superimposed with Gaussian noise with different noise levels were performed. The experiments indicate that misplacing the window that indicates which pixels contribute to the COG computation causes a bias and a larger variance in the estimated COG. This bias can be reduced by applying a threshold on the intensities comprised by the window. The chosen weighing scheme influences the accuracy and precision of the estimated COG, as thresholding results in a higher accuracy, but a worse precision. The desired trade-off between accuracy and precision can be chosen using the derived formula for the variance of the center of gravity. The difference between our estimate and the true variance was always less than $5 \%$ of the true variance and this deviation decreases with increasing signal-to-noise ratio. Our approximation to the estimate performed better than the one presented by Oron et al. [1] by up to a factor $\approx 10$ for a window misplacement of 3.0 pixels.
\end{abstract}

Keywords: center of gravity, centroid, variance, object position, object recognition, sub-pixel precision, measurement noise.

EDICS: 2-ANAL

\footnotetext{
${ }^{\dagger}$ Division of Image Processing (LKEB), Department of Radiology, Leiden University Medical Center, P.O.B. 9600, 2300 RC Leiden, The Netherlands, phone: +31-71-526.39.35, fax: +31-71-526.68.01.

1'e-mail address: h.c.van assen@lumc.nl, ${ }^{2}$ e-mail address: michael@cs.uu.nl, ${ }^{3}$ e-mail address: j.h.c.reiber@lumc.nl
} 


\section{INTRODUCTION}

Object detection plays an important role in many image processing problems. Examples from medical imaging are marker recognition [2, 3] and leukocyte tracking [4]. In remote sensing, tasks like automatic target recognition [57] and delineation of particular areas [8,9] are essential object recognition tasks. Also in radar imaging, objects like ships [10], oil spills [11] and synthetic objects [12] need to be detected automatically. In such applications, it is important to determine the position of each detected object with the highest possible accuracy and precision.

Detection of objects can be performed by approaches such as the Hough Transform [13], (non-) linear filters [14], or by pattern recognition techniques such as neural networks [3,4] or support vector machines [15]. Object recognition can also be performed by detecting the object boundaries, e.g., by dynamic programming [16] or snakes [17]. Most of these methods give as a result the most likely central pixel of each detected object, either directly by means of a filter response or indirectly by means of the centroid of all points on the boundary contour.

When the positions of the objects need to be known with sub-pixel precision, an accurate and robust estimate can be obtained by computing its center of gravity [18]. In case the recognition algorithm results in a binary output (pixel belonging to the object or not), the center of gravity of each object is reduced to computing the average coordinate along the ( $x, y$ and possibly $z$ ) axes among the pixels or voxels that are associated with the recognized object. When, on the other hand, the detection results in a set of output values distributed in a neighborhood around the object center (e.g., a filter response) and the output value is linearly related to the distance to the object center, the weighted center of gravity [18] may result in a more exact object location. Another application of the weighted center of gravity is in the computation of statistical moments such that the moments of $\mathrm{Hu}$ [19] and Zernike [20].

We will present a framework for analyzing the accuracy (bias) and precision (variance) of the weighted center of gravity in the presence of noise, see Fig. 1. For the weighted center of gravity, the distribution of the weights (intensities) among the pixels that belong to the object determines the accuracy of the center of gravity estimate as well as its precision as a function of the noise level. We use our framework to introduce a distinction between bias and variance. It is well known from the literature [21] that the weighted center of gravity may be a biased estimator for the true object center. We show - theoretically and through experiments - that the influence of background clutter on the calculation of the center of gravity of an object can be reduced by applying a threshold to a neighborhood that encompasses the detected object. This entails setting the weights associated with the gray values of all pixels of which the gray value is below some fixed value (the threshold) to zero, leaving all other gray values in the window intact. It is furthermore shown that application of a threshold may increase the accuracy of the estimated object center but that it can also lead to a larger variance, i.e., an increased sensitivity to noise. Moreover, the application of a threshold induces a discretization error, which biases the estimated center. The size of this bias depends on the threshold level and the intensity profile of the object under investigation [21].

In this paper, we first review different noise generating processes. Subsequently, we derive a novel formula for the variance of the weighted center of gravity measure as a function of the noise level in 2D and 3D images, along with two approximations of this formula. The validity of the approximative formulas and the circumstances under which these approximations suffice are investigated. Simulations illustrate the influence of the weighting scheme and the application of a threshold on the variance and bias of the calculated center of gravity. The applicability of the weighted center of gravity and its variance is illustrated by examples from calibration in radiographs.

In this paper, we first review different noise generating processes (section II). In section III, the center of gravity measure is introduced, while in section IV its variance is sub-divided into a bias of the mean and the variance around this mean center of gravity. Subsequently, we derive a novel formula for the variance of the weighted center of gravity measure along with two approximations. In section $\mathrm{V}$, the validity of the formulas and the circumstances under which these approximations suffice are investigated experimentally.

\section{BACKGROUND}

Define a noise-free image as a two-dimensional sampled function $F(x, y)$ of the discrete coordinates $x=1, \ldots, x_{\max }$ and $y=1, \ldots, y_{\max }$. Assume that the intensity of the pixels in a noisy image is the the result of successive realizations of a random variable $\varepsilon(x, y)$. The noise-generating process is defined by the class of stochastic functions $G: \Re^{1+d} \rightarrow \Re$

$$
f(x, y)=G(F(x, y), \boldsymbol{\theta})
$$

with $\boldsymbol{\theta}$ the $d$-dimensional parameter vector describing the stochastic model of the noise generating process, $G$. We distinguish between three (external) general noise generating processes:

- $\quad$ Additive noise, $f(x, y)=F(x, y)+\varepsilon(x, y)$

- Multiplicative noise, $f(x, y)=F(x, y) \cdot \varepsilon(x, y)$ [23]

- Impulsive (Salt-and-Pepper) noise,

$$
f(x, y)=F(x, y)+G(F(x, y), \theta)[22]
$$

The most simple stochastic process is that of additive noise. In fact, the linearity of the weighted center of gravity is justified in a situation with additive noise. Impulsive noise occurs in, e.g., transmission of images from remote satellites where the limited capacity of the very long communication lines has to be utilized optimally [24].

We demarcate our analysis of the weighted center of gravity to the situation with additive noise. Additive noise can be generated by several different underlying processes. A frequently occurring process in image formation is that of Gaussian noise, because according to the central limit theorem the average of many measurements under mild conditions converges towards the Gaussian distribution as their number approaches infinity. In radiography, the emission of electromagnetic radiation is characterized by a 
Poisson distribution [25] whereas, e.g., the reconstructed signals in Magnetic Resonance Images have a Rician distributed noise component [26]. Both the Poisson and the Rice distributions can be approximated by a Gaussian distribution; the approximations become better with a decreasing signal-to-noise ratio.

In the following, we assume that the noise in different pixels is uncorrelated. Pixels belonging to an object often have intensities, which are (inversely) proportional to their distance to the object center. Take as an example a convolution of an image with a linear filter of which the kernel resembles the object to be detected [3]. The convolution operation results in an output image in which the brightest spots indicate the most likely central coordinates of the objects to be detected. Fig. $2 b$ illustrates the output of a convolution with a kernel that resembles a radiopaque marker (Fig. 2a). Often, a threshold is applied to this output image and bright "islands" are taken to indicate detected objects (Fig. 2c).

\section{CENTER OF GRAVITY}

Let us define a detection function $d(x, y)$ (e.g., a filter), whose output is related to the probability that the coordinate $(x, y)$ is the central pixel of the object to be detected. In the sequel, we assume that the value $d(x, y) \propto$ $P($ center $=(x, y))$ for $(x, y)$ being in some neighborhood, $(x$ $y) \in \Omega$, in the vicinity of the true object center. In other words, it is only assumed that the detection algorithm results in a local maximum of $d(x, y)$ around the center of each detected object. If a global maximum were required, only objects with the same, maximal probability

$P(\operatorname{center}=(x, y))$ could be detected in an image whereas all objects with a lower output from the detection algorithm would be missed.

In a (two-dimensional) binary image, the binary center of gravity of the neighborhood $\Omega$ is defined as

$\varsigma(x, y)=\left(\frac{\sum_{x, y \in \Omega} x}{\operatorname{card}(\Omega)}, \frac{\sum_{x, y \in \Omega} y}{\operatorname{card}(\Omega)}\right)$

with $\Omega$ the (possibly connected) area containing True pixels which belong to the object to be detected, $\operatorname{card}(\Omega)$ denotes the number of True pixels in the neighborhood. In a graylevel image, the weighted center of gravity of the object is defined as [18]

$$
c(x, y)=\left(\frac{\sum_{x, y \in \Omega} x w(x, y)}{\sum_{x, y \in \Omega} w(x, y)}, \frac{\sum_{x, y \in \Omega} y w(x, y)}{\sum_{x, y \in \Omega} w(x, y)}\right)
$$

with $w(x, y)$ a function that weighs the coordinates defined as

$$
w(x, y)=a \cdot(f(x, y)-m)
$$

where $m<\min _{x, y \in \Omega}(f(x, y))$ when $a>0$ and $m>\max _{x, y \in \Omega}(f(x, y))$ when $a<0$. In the first case, where the parameter $a>0$, the center of gravity is attracted to the brighter pixels in $\Omega$ whereas for $a<0$, darker pixels attract $c(x, y)$.

In practical applications, $m=\min _{x, y \in \Omega}(f(x, y))-\delta, \delta>0$

(e.g. 0.01) and $a=1$ for bright-center objects, whereas $m=\max _{x, y \in \Omega}(f(x, y))+\delta, \delta>0$ and $a=-1$ for darkcenter objects.

It is clear that the binary center of gravity $\varsigma(x, y)$ is highly dependent on which pixels are regarded as belonging to the object. A proper identification of the local neighborhood of the object, $\Omega$, which is an indication for the placement of a window within which the centroid computation is performed, is crucial for the accuracy and precision of the estimated object center. The (estimated) binary center of gravity $\varsigma(x, y)$ is moreover sensitive to the chosen threshold value. The weighted center of gravity $c(x, y)$ is a remedy to this problem in the sense that the object centers estimated with Eq. (3) are less sensitive to the chosen threshold value, compare Figs. $2 \mathrm{c}$ and $2 \mathrm{~d}$. Application of a threshold also reduces the number of weights that contribute to the center of gravity estimate, but causes a larger variance. In fact, the choice of the threshold level imposes a trade-off between accuracy and precision, see Fig. 1.

The center of gravity results in a sub-pixel estimate of the central position of an object. However, working with sub-pixel precision can introduce the necessity of interpolation of gray values, because these are used as weights in the weighted center of gravity estimation. Interpolation entails smoothing and introduces errors into the final object detection result [21].

In the following, we will derive three estimators for the variance of the center of gravity. The variance is separated into a genuine variance term and a bias term.

\section{THE VARIANCE OF THE CENTER OF GRAVITY}

When the estimated center of gravity is seen as a realization of a noisy measurement, the level of the noise determines the uncertainty of the measured value. For the binary center of gravity $\varsigma(x, y)$, a variance formula has been derived in [27]. In the sequel, we derive a maximumlikelihood estimate for the variance of the weighted center of gravity as a function of the noise level in the image, $\sigma^{2}$.

Equation (3) shows that the noise is propagated to the weighted center of gravity through the weights $w(x, y)$.

However, both the numerator and the denominator in Eq.

(3) contain the same weight factors for each coordinate, so they are dependent stochastic variables.

Define the variance of the center of gravity for $n \rightarrow \infty$ as

$$
\begin{array}{r}
\operatorname{var}(c(x, y))=n^{-1}\left(\sum_{i=1}^{n}\left(c_{x}(x, y)-\gamma_{x}(x, y)\right)^{2},\right. \\
\left.\sum_{i=1}^{n}\left(c_{y}(x, y)-\gamma_{y}(x, y)\right)^{2}\right)
\end{array}
$$


with $\left(\gamma_{x}(x, y), \gamma_{y}(x, y)\right)$ denoting the true and $\left(c_{x}(x, y), c_{y}(\mathrm{x}, y)\right)$ the estimated center of gravity and $n$ the number of observations. We propose to divide the variance component of the weighted center of gravity into the variance of the estimates $c(x, y)$ in relation to the estimated mean $\bar{c}(x, y)$ and the (squared) bias component [28],

$(\bar{c}(x, y)-\gamma(x, y))^{2}$, which yields (see Appendix A)

$$
\begin{aligned}
& \operatorname{var}(c(x, y))= \\
& \left(n^{-1} \sum_{i=1}^{n}\left(c_{x}(x, y)-\bar{c}_{x}(x, y)\right)^{2}+\left(\bar{c}_{x}(x, y)-\gamma_{x}(x, y)\right)^{2},\right. \\
& \left.n^{-1} \sum_{i=1}^{n}\left(c_{y}(x, y)-\bar{c}_{y}(x, y)\right)^{2}+\left(\bar{c}_{y}(x, y)-\gamma_{y}(x, y)\right)^{2}\right)
\end{aligned}
$$

with

$$
\bar{c}(x, y)=\left(n^{-1} \sum_{i=1}^{n} c_{x}(x, y), n^{-1} \sum_{i=1}^{n} c_{y}(x, y)\right)
$$

The weighted center of gravity $c(x, y)$ is only an unbiased estimator of the true center $\gamma(x, y)$ when

$(\bar{c}(x, y)-\gamma(x, y))^{2} \rightarrow(0,0)$ for $n \rightarrow \infty$. On the other hand, the variance around the mean remains in the presence of noise. In the sequel, we derive a formula for $\operatorname{var}(x, y)$. Possible causes for a bias are investigated experimentally. We have identified three different causes for a biased center of gravity estimation:

- quantization bias

- discretization bias

- $\quad$ averaging bias

The quantization bias is caused by the sampling of the intensity function into a finite number of gray levels and is considered in detail by Morgan et al. [29]. The discretization bias is caused by sampling of the continuous spatial dimension into a discrete grid. This bias has been investigated thoroughly by Patwardhan [21]. The averaging bias is caused by misplacing the window $\Omega$ in relation to the underlying object. This bias will be explored experimentally in this paper.

In order to find an expression for the variance of the quotient of two stochastic terms, Eq. (3), we start off with the delta-method, which states that for a stochastic variable $Z[30]$

$$
\operatorname{var}(\mathrm{Z}) \approx \operatorname{var}(\ln (\mathrm{Z})) \cdot \mu_{\mathrm{Z}}^{2}
$$

Let $Z$ denote the quotient of two entities $X$ and $Y$ and $\mu_{Z}$ its mean, so Eq. (8) can be rewritten as

$$
\begin{aligned}
\operatorname{var}\left(\frac{X}{Y}\right) \approx & \operatorname{var}\left(\ln \left(\frac{X}{Y}\right)\right) \cdot\left(\frac{\mu_{X}}{\mu_{Y}}\right)^{2}= \\
& \operatorname{var}(\ln (X)-\ln (Y)) \cdot\left(\frac{\mu_{X}}{\mu_{Y}}\right)^{2}= \\
& (\operatorname{var}(\ln (X))+\operatorname{var}(\ln (Y))- \\
& 2 \cdot \operatorname{cov}(\ln (X), \ln (Y))) \cdot\left(\frac{\mu_{X}}{\mu_{Y}}\right)^{2}
\end{aligned}
$$

Applying Eq. (8) to each of the terms in Eq. (9) yields

$$
\begin{aligned}
\operatorname{var}\left(\frac{X}{Y}\right) \approx & \left(\frac{\operatorname{var}(X)}{\mu_{X}{ }^{2}}+\frac{\operatorname{var}(Y)}{\mu_{Y}{ }^{2}}-\right. \\
& \left.2 \cdot \frac{\operatorname{cov}(X, Y)}{\mu_{X} \mu_{Y}}\right) \cdot\left(\frac{\mu_{X}}{\mu_{Y}}\right)^{2}
\end{aligned}
$$

It should be noticed that Eq. (10) is based on the deltamethod and as such remains an approximation to $\operatorname{var}(X / Y)$. The approximation holds well when all observations of the stochastic variables $X$ and $Y$ are positive and their first two moments are well-defined. Moreover, the coefficients of variation of $X$ and $Y$ and $X / Y$ should be less than 1, where the coefficient of variation (CV) of $X$ is defined as

$\mathrm{CV}(X)=\sqrt{\frac{\operatorname{var}(X)}{\mu_{X}{ }^{2}}}$

Now we define $X$ as the numerator and $Y$ as the denominator in Eq. (3)

$$
\begin{aligned}
& X(\Omega)=\left(\sum_{x, y \in \Omega} x w(x, y), \sum_{x, y \in \Omega} y w(x, y)\right), \\
& Y(\Omega)=\sum_{x, y \in \Omega} w(x, y)
\end{aligned}
$$

Inserting Eq. (12) and (13) into Eq. (10) yields

$$
\begin{aligned}
& \operatorname{var}(c(x, y)) \approx \\
& \int\left(\frac{\operatorname{var}\left(\sum_{x, y \in \Omega} x w(x, y)\right)}{\mu_{x, y \in \Omega} \sum_{x, y}{ }^{2}}+\frac{\operatorname{var}\left(\sum_{x, y \in \Omega} w(x, y)\right)}{\mu_{\substack{\left.\sum, y \in \Omega \\
x, y, y\right)}}{ }^{2}}-\right.
\end{aligned}
$$

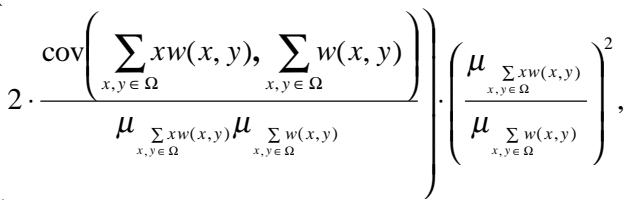

$$
\begin{aligned}
& \left(\frac{\operatorname{var}\left(\sum_{x, y \in \Omega} y w(x, y)\right)}{\mu_{x, y \in \Omega} \sum_{x,(x, y)}{ }^{2}}+\frac{\operatorname{var}\left(\sum_{x, y \in \Omega} w(x, y)\right)}{\mu_{\substack{\sum \\
x, y \in \Omega}}{ }^{2}}-\right.
\end{aligned}
$$

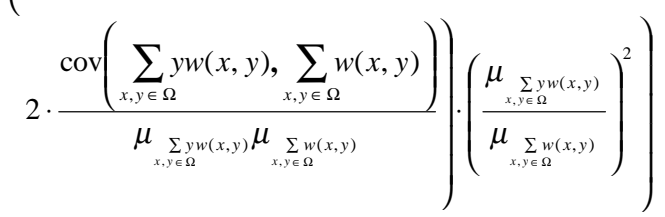

Because the centers of the $x$ - and $y$-coordinates are independent, we will continue the derivation for the $x$ coordinate only; for the $y$-coordinate, the same derivation will hold. 
Above, we defined $\varepsilon(x, y)$ in Eq. (1) as a normally distributed random variable, $\varepsilon(x, y) \sim g\left(0, \sigma^{2}\right)$. From this definition and from the part in Eq. (12) related to the $x$ coordinate, we can estimate the average and variance in $X$ as [30]

$$
\begin{aligned}
& \hat{\mu}_{X}(\Omega) \approx N \cdot \hat{\mu}_{w} \cdot \hat{x}_{C O G} \\
& \hat{\sigma}_{X}^{2}(\Omega)=\sigma^{2} \cdot \sum_{x, y \in \Omega} x^{2}
\end{aligned}
$$

with $N=\operatorname{card}(\Omega), \hat{\mu}_{w}$ the estimated average of the weights in $\Omega$, and $\hat{x}_{C O G}$ the coordinate of the estimated center of gravity along the $x$ axis, in the neighborhood $\Omega$.

Similarly, the average and variance of the denominator, $Y$, in Eq. (13) become

$$
\hat{\mu}_{Y}=N \cdot \hat{\mu}_{w}
$$

$\hat{\sigma}_{Y}^{2}=N \cdot \sigma^{2}$

Let the center of the area $\Omega$ be the origin of the coordinate system such that the covariance term in Eq. (10) can be omitted [1]. This yields the following approximation to the variance of the center of gravity

$$
\begin{aligned}
& \operatorname{var}\left(\left(\frac{c(x, y)) \approx}{\left(N \cdot \hat{\mu}_{w} \cdot \hat{x}_{C O G}\right)^{2}}+\frac{\sigma^{2}}{N \cdot \hat{\mu}_{w}{ }^{2}}\right) \cdot \hat{x}_{C O G}{ }^{2},\right. \\
& \left.\left(\frac{\sigma^{2} \cdot \sum_{x, y \in \Omega} y^{2}}{\left(N \cdot \hat{\mu}_{w} \cdot \hat{y}_{C O G}\right)^{2}}+\frac{\sigma^{2}}{N \cdot \hat{\mu}_{w}{ }^{2}}\right) \cdot \hat{y}_{C O G}{ }^{2}\right)
\end{aligned}
$$

If the object is positioned centrally inside the neighborhood $\Omega$ of the marker's center of gravity (i.e., $x_{C O G}$ and $y_{C O G}$ are very close to zero) the second terms inside the

brackets in Eq. (19) (i.e. $\sigma^{2} / N \cdot \hat{\mu}_{w}{ }^{2}$ ) can also be neglected

and, thus, Eq. (14) simplifies to

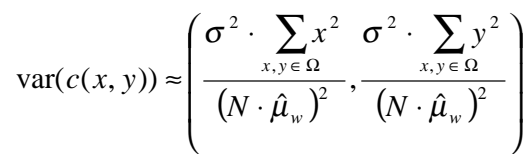

which is the approximation derived by Oron et al. [1]. The two variance Eqs. (19) and (20) will be compared experimentally.

\section{EXPERIMENTS}

Simulations with synthetic objects were carried out to investigate the accuracy of the derived variance estimators, Eqs. (10), (19) and (20). The effect of applying a threshold to the noisy object images before computing the center of gravity was investigated as well.

\section{A. Experimental set-up}

We conducted a number of simulations in which 2,500 markers with a circular (in $x$ and $y$ ) cosine shape [3] and a size of $10 \times 10$ pixels were generated (Fig. 3a). The gray values of the centers of the markers were set close to 2 whereas the values at the edges were close to 0 . Gaussian noise with a zero mean and a standard deviation ranging from $\sigma=0.0$ to $\sigma=0.50$, with steps of 0.05 was superimposed on the marker images (Fig. 3b). Five experiments using a 10x10-sized window as the neighborhood $\Omega$ were performed. The centers of gravity of the markers in the first three experiments were translated by $\Delta x \in\{0,0.5,1.0,1.5,2.0\}$ pixels from the center of the window $\Omega$, before adding the Gaussian noise. In the fourth experiment, the marker centers were shifted by $\Delta x \in\{0$, $1.5,3.0\}$ pixels.

Additionally, a sixth experiment was performed in which a radiograph of a rectangular calibration grid was used to study image deformations. The distances between crossing points spanned by the horizontal and vertical cords on the grid were computed in order to decide whether de-warping the images makes sense in the presence of pincushion distortion. For all experiments, the conditions next to Eq. (10) were met.

\section{B. Experiment $1-$ Misplacing the window}

In the first experiment, the influence of translating the object away from the center of the window (being 5.5) on the center of gravity measure was investigated (bias due to window misplacement). Figure 4a shows the results from the first experiment. The weighted center of gravity has an intrinsic bias towards the center of the window. Adding noise to the objects increases this bias towards the center of the window $\Omega$. Consequently, the shape of the objects has less influence for an increasing amount of noise. Figure $4 \mathrm{~b}$ shows that applying a threshold to the marker images before calculating the centers of gravity reduces the bias. This improvement is caused by excluding pixels that do not belong to the marker. The threshold was applied such that the center of gravity was always based on the 16 largest gray values in the window $\Omega$, approximately the size of the marker. It is clear from this experiment that the bias caused by misplacing the window can be reduced by application of a threshold.

\section{Experiment 2 - Variance of the center of gravity}

In the second experiment, the influence of adding noise to the markers on the variance of the center of gravity was investigated. The variance in the center of gravity was calculated directly from the simulated data and also estimated by Eq. (14). The markers were translated within the window along the $x$-direction as was described in experiment 1 . The results of the second experiment show that the relation between the variance of the calculated centers of gravity and the standard deviation of the noise in the marker images is almost linear (Figure 5a). Figure 5b shows that the true variance and the variance estimated by Eq. (14) deviate less than $2.5 \%$ o. So the approximation in Eq. (14) is almost exact. Translating the markers within the 
window results in larger variances of the center of gravity. This translation introduces areas with lower average gray values into the window but with the same noise component This explains the deviating curves in Fig. 5a. Thus, pixels with a high SNR are replaced with pixels with a low SNR, which enlarges the resulting variance in the estimated center of gravity.

\section{Experiment 3 - Variance of the center of gravity with threshold}

The purpose of the third experiment was twofold. First, we investigated the effect thresholding has on the variance of the estimated center of gravity. Secondly, we studied the behavior of the variance estimator when translating the true object center. This experiment was similar to the second one, except from the fact that a threshold was applied to each marker image before calculating the center of gravity and estimating its variance. The threshold was applied such that the center of gravity was always based on the 16 largest gray values in the window $\Omega$. The remaining weights were set to zero.

This experiment shows the relation between the application of the threshold and the increasing dispersion in the resulting center of gravity measures as a function of a poorer signal-to-noise ratio. An almost linear relation is seen between the variance of the calculated centers of gravity and the standard deviation of the noise in the marker images (see Fig. 5c). However, the order of magnitude of the resulting variances in the centers of gravity is approximately twenty times as large as those observed in the second experiment (see Fig. 5a). This is the result of the smaller number of pixels $(\mathrm{N}=16)$ that contribute to the center of gravity calculation as compared to the set-up in the second experiment $(\mathrm{N}=100)$.

The effect of translating the object center - with and without application of a threshold - is clearly visible by comparing Fig. 5a and 5c. Noticing the difference in the order of magnitude of the y-axes in the two figures, it is apparent that the application of a threshold implies that the variance becomes independent of the position of the true object center with respect to the window (Fig $5 \mathrm{c}$ ). When this threshold is not applied, the variance of the center of gravity becomes dependent of the object center. Consequently, the certainty of the estimated object center will depend on where in the window the object is positioned.

The differences between the estimated (Eq. (14)) and true variances increase with the amount of noise in the marker images, but they are always less than $5 \%$ of the true variance (Figures $5 \mathrm{c}$ and $5 \mathrm{~d}$ ).

\section{E. Experiment 4 - Precision of the approximative variance estimators}

In the fourth experiment, the true variance in the center of gravity measures was compared to the approximations from Eqs. (19) and (20). Again, 2,500 markers were generated and superimposed with Gaussian noise. The markers were similar to those used in the previous three experiments, also the same noise levels were used. No threshold was applied in this experiment, but the markers were translated along the $x$-direction. The variances estimated with the Eqs. (19) and (20) were compared with the true variance, for markers that were translated by $0,1.5$ and 3.0 pixels along the $x$-direction.

The simulations show that Eq. (19) is a good

approximation to the true resulting variance of the center of gravity measure (Figure 6a), and Eq. (20) is a good approximation only if the marker is positioned centrally in the window. For situations where the true center of gravity differs from the center of the window (see Figures $6 \mathrm{~b}$ and 6c) the results from Eq. (20) deviate considerably from the true variances. From this experiment, it is clear that Eq. (19) outperforms Eq. (20), the approximation derived by Oron et al. [1]. The poorer performance of Eq. (20) is a result of ignoring the second term (i.e. $\left.\operatorname{var}(Y) / \mu_{Y}{ }^{2}\right)$ in Eq. (10). Additional simulations indicated that if the origin of the coordinate system was not positioned in the expected center of gravity, both Eqs. (19) and (20) result in biased variance estimates. This can be explained by the fact that in Eqs. (19) and (20), the variance depends on the coordinates in the neighborhood of the object. Equations (19) and (20) require the origin of the coordinate system to be placed in the center of gravity of the marker. Additionally, Eq. (20) requires the center of the window to be positioned in the center of gravity of the marker. Both requirements entail pixel interpolation. The actual variance in the center of gravity does not depend on how the coordinate system is positioned. The covariance term in the almost exact formula Eq. (10) accounts for the translation of the origin.

\section{F. Experiment 5 - Inversion of intensity distributions}

In the fifth experiment, we investigated the influence of the value of $m$ in Eq. (4) on the center of gravity measure and its variance, without translating the markers within the window. In this experiment, sets of 2,500 markers, similar to those used in the first experiments were generated, but the markers were inverted, so the center of the marker was the minimum and the peaks were on the verge between marker and background (like the situation sketched in Fig. 2a). The marker intensities still ranged from 0 to 2, before noise was added. The value of $m$ ranged from 2.3 to 15 , but was always chosen such that $m>\max _{x, y \in \Omega}(f(x, y))$ with $f(x, y)$ the intensity of a marker image at coordinate $(x, y)$.

The maximum gray values within the window ranged from 2.0 for the markers without noise to 3.95 for the markers with noise added with a standard deviation of 0.50 . The results indicate that for all noise levels, increasing the value of $m$ biases the center of gravity measure towards the center of the window (Fig. 7a). Figure 7b shows that the variance in the center of gravity decreases (for all noise levels) as a function of $m$. According to Figs. $7 \mathrm{a}$ and $7 \mathrm{~b}$, a larger value of $m$ (with $a<0$ ) causes the influence on the $\mathrm{COG}$ of the gray values in the window $\Omega$ in Eq. (4) to decrease, i.e., $m$ operates as a smoothing operator besides inverting the intensity distribution. That explains why the center of gravity moves towards the center of the window with an increasing value of $m$ (with $a<0$ ). Moreover, a larger value of $m$ results in a smaller variance in the center of gravity measure. For very large values of $m$, the variance in the center of gravity tends to zero. In order to minimize the influence of the parameter $m$, its value should be chosen 
close to the largest gray value, always applying the conditions specified in relation to Eq. (4) for $m$.

\section{G. Experiment 6-Classification of image deformation}

In this experiment, we used a radiograph of a rectangular calibration grid (see Fig. 8a.) to determine the amount of image deformation caused by e.g. pincushion distortion. An image of the grid was acquired with a tube voltage of 60 $\mathrm{kV}$. This image was convolved with the LoG (Laplacian of the Gaussian kernel) [32] in order to find the crossings of the horizontal and vertical bands, see Fig. 8b. The crossings appear as dots in the result image, from which their locations were estimated using the Hough Transform. Precise locations for the band crossings were calculated using the center of gravity formula, Eq. (3).

Before calculating each center of gravity from the radiograph itself, the neighborhood $\Omega$ was superimposed with additive Gaussian noise of specific noise levels, $\sigma$. The resulting signal-to-noise-ratios (SNR) were $12.3 \mathrm{~dB}$, $18.3 \mathrm{~dB}$ and $24.3 \mathrm{~dB}$, respectively, and $\infty$, when no noise was superimposed.

For all situations, we calculated three horizontal and three vertical distance cords between designated pairs of crossings. The length of each cord was the average of 10 repeated measurements, see Fig. 8 b.

The variance in the resulting distances was calculated from the variances in the centers of gravity for the crossings, which were estimated using Eq. (19). We assumed that the noise we superimposed on the crossing areas was uncorrelated. Consequently, the variance of each cord length was calculated as the sum of the variances in two crossings which span the cord.

Finally, using Student's T-test, we tested the hypothesis $\mathrm{H}_{0}$ that the parallel (vertical or horizontal) cords have the same length

For the cases with a SNR of $\infty$ this test could not be performed, because the resulting estimated variances in the measured distances were zero.

Table I shows the test results for the hypothesis $\mathrm{H}_{0}$ : two parallel cords have the same length. We used the critical value for Student's double-sided T-test with $\alpha=0.05$ and the degrees of freedom $v=18$ ( 2 times 10 repetitions minus 1 ) which is 2.101. From Table I, it appears that when the SNR deteriorates the number of significantly different cord lengths decreases, i.e., the hypothesis $\mathrm{H}_{0}$ cannot be rejected for an increasing number of distance pairs. This means that with a higher amount of noise, it becomes increasingly difficult to determine whether local image deformation is present. This experiment shows an application of our variance estimator Eq. (19).

\section{DISCUSSION}

We investigated the accuracy and precision of the estimated center of gravity of detected objects (e.g. radiopaque markers or leukocytes) in an image as estimate for the central object position. Initial experiments indicated that thresholding should be applied when the window from which the object center is computed, is translated in relation to the true object center. Without previous thresholding, the computed center of gravity becomes a biased estimate of the true center.

We derived three formulas for the variance of the center of gravity estimate as a function of the signal-to-noise ratio in the image, Eq. (14), Eq. (19) and Eq. (20), respectively. Our simulations indicate that all three give good variance estimates when the window is positioned centrally on the marker. When the true marker center is translated in relation to the center of the window, before the center of gravity is estimated, the approximation derived by Oron et al. (Eq. (20)) [1] underestimates the true variance of the center of gravity significantly. Hence, Eq. (20) is a biased estimator of the variance in the center of gravity. Our more exact approximation, Eq. (19), estimates the true variance within a margin of $8 \%$ for a marker shift with respect to the window center $\Delta x=0$, a margin of $5.7 \%$ for $\Delta x=1.5$ and of $4.2 \%$ for $\Delta x=3.0$. For $\Delta x=0$, Eqs. (19) and (20) give the same results; for $\Delta x=1.5$, Eq. (20) estimates the true variance within a margin of $24 \%$ and for $\Delta x=3.0$ within a margin of $52 \%$.

Both the estimated center of gravity and its variance depend on the size of the window that encompasses the pixels that are regarded as belonging to the object. It is clear from both Eq. (19) and Eq. (20) that a larger $N$ leads to a decrease in the variance as $N$ occurs in the denominators of both variance formulas. Decreasing the size of the window, on the other hand, diminishes the probability that pixels that do not belong to the particular object enter the calculation. Hence, the center of gravity may be estimated more accurately, because this decreases the averaging bias.

As already stated, our simulations indicate that a threshold should in general be applied. Several automatic methods have been developed for optimal threshold selection, for a discussion, see e.g. [33]. Which threshold value is optimal for estimating the center of gravity, depends on the intensity pattern surrounding the object center, the chosen window size, the level of the noise present and the preferred trade-off between the (inversely related) averaging bias and discretization bias, and variance. A high threshold implies that only a few pixels enter the computation. A low threshold results in more averaging, because more pixels enter the center-of-gravity calculation, such that noise has less influence on the center of gravity estimate. However, this compromises the accuracy (averaging bias) in the sense that the center of gravity estimate becomes biased by the positioning of the window. Conclusively, both the choice of the optimal window size and threshold value entail a trade-off between bias (averaging over many pixels, discretizing the underlying continuous signal) and variance (including only a few pixels in the computation).

The theoretical results and the results obtained from the simulation experiments can be summarized in table form. Table II indicates the effects of varying parameters such as window size, shifting the object, increasing the noise level and the application of a threshold on the bias and variance of the estimated center of gravity. Patwardhan [21] showed that the application of a threshold to the neighborhood $\Omega$ introduces a discretization bias, the size of which depends on the radius of the object and the number of pixels that contribute to the center of gravity estimate. So, changing 
the threshold level causes coordinates to be used or discarded in the center of gravity computation.

Consequently, the discrepancy between the continuous and the discrete center of gravity shows a jagged pattern when the object is shifted with sub-pixel precision. The discretization bias cannot exceed 0.5 pixel assuming a connection criterion is applied. The discretization bias decreases when more pixels enter the center of gravity computation. So, lowering the threshold leads to a decrease in the discretization bias (see Table II).

To avoid errors caused by window misplacement, an iterative center of gravity calculation could be used, relocating the window between iterations. As always, an iterative approach brings about the subject of convergence.

This could be a subject for further research.

In the case that the center of gravity of an elongated object (which is non-isotropic) is to be determined, different optimal window sizes in the $x$ - and $y$-directions apply. As a consequence, the trade-offs between bias and variance are different for the two directions. The number of contributing pixels will be the same for the calculation of the center of gravity estimates for all directions, i.e., the number $N$ of pixels inside the window $\Omega$. Assuming that the variance in the underlying coordinate distribution is larger in the direction in which the object is larger, the resulting variance in the center of gravity and its estimates will also be larger in this direction.

The center of gravity, Eq. (3), and variance formula, Eq. (19), can easily be extended to 3-dimensions which can be useful when determining object centers in, e.g., CT- and MR-images. The window $\Omega$ becomes 3-dimensional, resulting in the following center of gravity formula

$$
c(x, y, z)=\left(\begin{array}{l}
\frac{\sum_{x, y, z \in \Omega} x w(x, y, z)}{\sum_{x, y, z \in \Omega} w(x, y, z)}, \\
\sum_{x, y, z \in \Omega} y w(x, y, z) \\
\\
\sum_{x, y, z \in \Omega} w(x, y, z) \\
\sum_{x, y, z \in \Omega} z w(x, y, z) \\
\sum_{x, y, z \in \Omega} w(x, y, z)
\end{array}\right)
$$

and variance

$$
\begin{aligned}
& \operatorname{var}(c(x, y, z)) \approx \\
& \left(\left(\frac{\sigma^{2} \cdot \sum_{x, y, z \in \Omega} x^{2}}{\left(N \cdot \hat{\mu}_{w} \cdot \hat{x}_{C O G}\right)^{2}}+\frac{\sigma^{2}}{N \cdot \hat{\mu}_{w}{ }^{2}}\right) \cdot \hat{x}_{C O G}{ }^{2},\right. \\
& \left(\frac{\sigma^{2} \cdot \sum_{x, y, z \in \Omega} y^{2}}{\left(N \cdot \hat{\mu}_{w} \cdot \hat{y}_{C O G}\right)^{2}}+\frac{\sigma^{2}}{N \cdot \hat{\mu}_{w}{ }^{2}}\right) \cdot \hat{y}_{C O G}{ }^{2}, \\
& \left.\left(\frac{\sigma^{2} \cdot \sum_{x, y, z \in \Omega} z^{2}}{\left(N \cdot \hat{\mu}_{w} \cdot \hat{z}_{C O G}\right)^{2}}+\frac{\sigma^{2}}{N \cdot \hat{\mu}_{w}{ }^{2}}\right) \cdot \hat{z}_{C O G}{ }^{2}\right)
\end{aligned}
$$

as based on Eq. (19).

We investigated the influence of Gaussian distributed additive "white" noise on the accuracy and precision of the estimated center of gravity. The noise inherent in some imaging modalities is known not to be Gaussian. An example is radiography where the number of particles in an $\mathrm{X}$-ray emitted in the time interval during exposure follows a Poisson distribution. In many cases, the Gaussian distribution will be a sufficient approximation for the slightly skew Poisson distribution.

With the last experiment we showed that our variance estimator in Eq. (19) can be applied to detect image deformations in the presence of noise.

\section{CONCLUSION}

In this paper, we have studied the behavior of the (weighted) center of gravity measure as a function of additive noise present in the gray value image. Furthermore, we analyzed the influence of applying a threshold to the gray value image (which determines the weighing scheme) for a possible bias and variance of the center of gravity measure. The decision whether to apply a threshold to the gray value image or not, is basically a choice between accuracy and precision. Application of a threshold before the center of gravity is computed, results in general in a better accuracy (smaller bias) but a worse precision. For a specific application where the exact object centers should be known, we recommend that experiments are being conducted as to establish the desired trade-off between bias and variance. The factors that should be varied are listed in Table II.

We have compared two formulas for the estimation of the variance of the center of gravity measure with the true variance. Our approximation (Eq. (19)) performs much better than the one presented by Oron [1] (Eq. (20)) when the evaluation window $\Omega$ is not centered on the true center of gravity. Because the true center of gravity is generally unknown beforehand, a misplacement of the evaluation window $\Omega$ might easily occur. In this case, if an estimation of the precision of the center of gravity measure is requested, our novel estimator (Eq. (19)) should be applied.

When an image is affected by multiplicative noise, the natural logarithm should be applied on the image, before the center of gravity is computed. The natural logarithm operation converts multiplicative noise to additive noise, for which our formulas hold.

It is clear that caution should be taken with respect to how the pixel intensities are weighed. The condition next to Eq. (4) has to be obeyed to avoid weights smaller than or equal to zero. A weight equal to zero means that the corresponding coordinate is excluded from the center of gravity calculation; negative weights cause the center of gravity to be pulled in the wrong direction. Both situations cause a bias in the resulting center of gravity measure and therefore have to be avoided.

In conclusion, we can state that in order to find the best estimate for the center of gravity in a gray level image, a threshold should in general be applied to (the local neighborhood in) the image before calculating a center of 
gravity measure. Our variance formula makes it possible to estimate the trade-off between accuracy and precision, in the presence of noise.

\section{APPENDIX A}

In section IV, we define the variance of the center of gravity for $n \rightarrow \infty$ as

$$
\begin{array}{r}
\operatorname{var}(c(x, y))=n^{-1}\left(\sum_{i=1}^{n}\left(c_{x}(x, y)-\gamma_{x}(x, y)\right)^{2},\right. \\
\left.\sum_{i=1}^{n}\left(c_{y}(x, y)-\gamma_{y}(x, y)\right)^{2}\right)
\end{array}
$$

with $\left(\gamma_{x}(x, y), \gamma_{y}(x, y)\right)$ denoting the true and $\left(c_{x}(x, y), c_{y}(\mathrm{x}, y)\right)$ the estimated center of gravity and $n$ the number of observations. Next, we propose to divide the variance component of the weighted center of gravity into the variance of the estimates $c(x, y)$ in relation to the estimated mean $\bar{c}(x, y)$ and the (squared) bias component [28], $(\bar{c}(x, y)-\gamma(x, y))^{2}$.

In order to achieve this, we add (only $x$-components are shown, $y$-components are identical) $\bar{c}_{x}(x, y)$ to the term $\left(c_{x}(x, y)-\gamma_{x}(x, y)\right)^{2}$ in Eq.(5). This yields

$$
\begin{array}{r}
\operatorname{var}_{x}(c(x, y))=n^{-1} \sum_{i=1}^{n}\left(c_{x}(x, y)-\bar{c}_{x}(x, y)+\right. \\
\left.\bar{c}_{x}(x, y)-\gamma_{x}(x, y)\right)^{2}
\end{array}
$$

Expanding the quadratic term, gives us

$$
\begin{array}{r}
\operatorname{var}_{x}(c(x, y))=n^{-1} \sum_{i=1}^{n}\left(c_{x}(x, y)-\bar{c}_{x}(x, y)\right)^{2}+ \\
n^{-1} \sum_{i=1}^{n}\left(\bar{c}_{x}(x, y)-\gamma_{x}(x, y)\right)^{2}- \\
2 n^{-1} \sum_{i=1}^{n}\left(c_{x}(x, y)-\bar{c}_{x}(x, y)\right) . \\
\left(\bar{c}_{x}(x, y)-\gamma_{x}(x, y)\right)
\end{array}
$$

In this last equation (Eq. (A.2)) the term $\left(\bar{c}_{x}(x, y)-\gamma_{x}(x, y)\right)$ is a constant and because of the definition of $\bar{c}(x, y)$ in Eq.

(7) the term $n^{-1} \sum_{i=1}^{n}\left(c_{x}(x, y)-\bar{c}_{x}(x, y)\right)$ is equal to zero.

Substituting this finding in Eq. (A.2), results in

$$
\begin{aligned}
\operatorname{var}_{x}(c(x, y))= & n^{-1} \sum_{i=1}^{n}\left(c_{x}(x, y)-\bar{c}_{x}(x, y)\right)^{2}+ \\
& n^{-1} \sum_{i=1}^{n}\left(\bar{c}_{x}(x, y)-\gamma_{x}(x, y)\right)^{2}
\end{aligned}
$$

which is equal to the $x$-component of Eq. (6).

\section{REFERENCES}

[1] E. Oron, A. Kumar, and Y. Bar-Shalom, "Precision tracking with segmentation for imaging sensors," IEEE Transactions on Aerospace and Electronic Systems, vol. 29, pp. 977-987, 1993.

[2] H. C. Van Assen, H. A. Vrooman, M. Egmont-Petersen, J. G. Bosch, G. Koning, E. L. Van Der Linden, B. Goedhart, and J. H. C. Reiber, "Automated Calibration in Vascular X-Ray Images Using the Accurate Localization of Catheter Marker Bands," Investigative Radiology, vol. 35, pp. 219-226, 2000.

[3] M. Egmont-Petersen and T. Arts, "Recognition of radiopaque markers in X-ray images using a neural network as nonlinear filter," Pattern Recognition Letters, vol. 20, pp. 521-533, 1999.

[4] M. Egmont-Petersen, U. Schreiner, S. C. Tromp, T. M. Lehmann, D. W. Slaaf, and T. Arts, "Detection of leukocytes in contact with the vessel wall from in vivo microscope recordings using a neural network," IEEE Trans. Biomed. Eng., vol. 47, pp. 941-951, 2000.

[5] V. Z. Këpuska and S. O. Mason, "A hierarchical neural network system for signalized point recognition in aerial photographs," Photogrammetric Engineering \& Remote Sensing, vol. 61, pp. 917-925, 1995.

[6] M. W. Roth, "Survey of neural network technology for automatic target recognition," IEEE Transactions of Neural Networks, vol. 1, pp. 28-43, 1990.

[7] A. M. Waxman, M. C. Seibert, A. Gove, D. A. Fay, A. M. Bernardon, C. Lazott, W. R. Steele, and R. K. Cunningham, "Neural processing of targets in visible multispectral IR and SAR imagery," Neural Networks, vol. 8, pp. 1029-1051, 1995.

[8] J. Desachy, L. Roux, and E.-H. Zahzah, "Numeric and symbolic data fusion: A soft computing approach to remote sensing images analysis," Pattern Recognition Letters, vol. 17, pp. 1361-1378, 1996.

[9] P. P. Raghu and B. Yegnanarayana, "Multispectral image classification using gabor filters and stochastic relaxation neural network," Neural Networks, vol. 10, pp. 561-572, 1997.

[10] C. Alippi, "Real-time analysis of ships in radar images with neural networks," Pattern Recognition, vol. 28, pp. 1899-1913, 1995.

[11] T. Ziemke, "Radar image segmentation using recurrent artificial neural networks," Pattern Recognition Letters, vol. 17 , pp. 319-334, 1996.

[12] J. C. Principe, M. Kim, and J. W. Fisher, "Target discrimination in synthetic aperture radar using artificial neural networks," IEEE Transactions on Image Processing, vol. 7, pp. 1136-1149, 1998.

[13] P. V.C. Hough, "A method and means for recognizing complex patterns," in US Patent Application No. 3069654. USA, 1962.

[14] H. Sjoberg, F. Goudail, and P. Refregier, "Comparison of the maximum likelihood ratio test algorithm and linear filters for target location in binary images," Optics Communications, vol. 163, pp. 252-258, 1999.

[15] M. Pontil and A. Verri, "Support Vector Machines for 3D object recognition," IEEE Transactions on Pattern Analysis and Machine Intelligence, vol. 20, pp. 637646, 1998.

[16] J. J. Gerbrands, "Segmentation of noisy images," in Information Technology and Systems. Delft: University of Technology Delft, 1988, pp. 170. 
[17] M. Kass, A. Witkin, and D. Terzopoulos, "Snakes: Active contour models," International Journal of Computer Vision, vol. 13, pp. 321-331, 1987.

[18] R. C. Gonzalez and R. E. Woods, Digital Image Processing. Reading, MA: Addison-Wesley, 1992.

[19] M. K. Hu, "Visual pattern recognition by moment invariants," IRE Transactions on Information Theory, vol. 8, pp. 179-187, 1962.

[20] F. Zernike, "Beugungstheorie des Schneidenverfahrens und seiner verbesserten Form der

Phasenkontrastmethode," Physica, vol. 1, pp. 689-701, 1934.

[21] A. Patwardhan, "Subpixel position measurement using 1D, 2D and 3D centroid algorithms in confocal microscopy," Journal of Microscopy, vol. 186, pp. 246257, 1997.

[22] E. M. Eliason and A. S. McEwan, "Adaptive box filters for removal of random noise from digital images," Photogrammetric Engineering and Remote Sensing, vol. 56, pp. 453-458, 1990.

[23] B. Aiazzi, L. Alparone, and S. Baronti, "Multiresolution local-statistics specle filtering based on a ratio laplacian pyramid," IEEE Transactions on Geoscience and Remote Sensing, vol. 36, pp. 1466-1476, 1998.

[24] E. M. Eliason and A. S. McEven, "Adaptive box filters for removal of random noise from digital images," Photogrammetric Engineering and Remote Sensing, vol. 56, pp. 453-458, 1990.

[25] Encyclopaedia Britannica. Chicago: Encyclopaedia Britannica Inc., 1997.

[26] J. Sijbers, A. J. den Dekker, P. Scheunders, and D. Van Dyck, "Maximum-likelihood estimation of Rician distribution parameters," IEEE Transactions on Medical Imaging, vol. 17, pp. 357-361, 1998.

[27] A. Kumar, Y. Bar-Shalom, and E. Oron, "Precision tracking based on segmentation with optimal layering for imaging sensors," IEEE Transactions on Pattern Analysis and Machine Intelligence, vol. 17, pp. 182188, 1995.

[28] M. P. Wand and M. C. Jones, Kernel smoothing. London: Chapman \& Hall, 1995.

[29] J. S. Morgan, D. C. Slater, J. G. Timothy, and E. B. Jenkins, "Centroid position measurements and subpixel sensitivity variations with the MAMA detector," Applied Optics, vol. 28, pp. 1178-1192, 1989.

[30] C. Cox, "Delta Method," in Encyclopedia of biostatistics, vol. 2, P. Armitage and T. Colton, Eds. Chichester, England: John Wiley \& Sons, 1998, pp. 1125-1127.

[31] M. B. Priestley, Spectral analysis and time series, 7 ed. London: Academic Press Limited, 1992.

[32] B. M. Dawant and A. P. Zijdenbos, "Image Segmentation," in Medical Imaging, vol. 2, M. Sonka and J. M. Fitzpatrick, Eds. Bellingham, Washington: SPIE - The International Society for Optical Engineering, 2000, pp. 71-127.

[33] J.-S. Chang, H.-Y. M. Liao, M.-K. Hor, J.-W. Hsieh, and M.-Y. Chern, "New automatic multi-level thresholding technique for segmentation of thermal images," Image and Vision Computing, vol. 15, pp. 2334, 1997. 


\section{FIGURES AND TABLES}

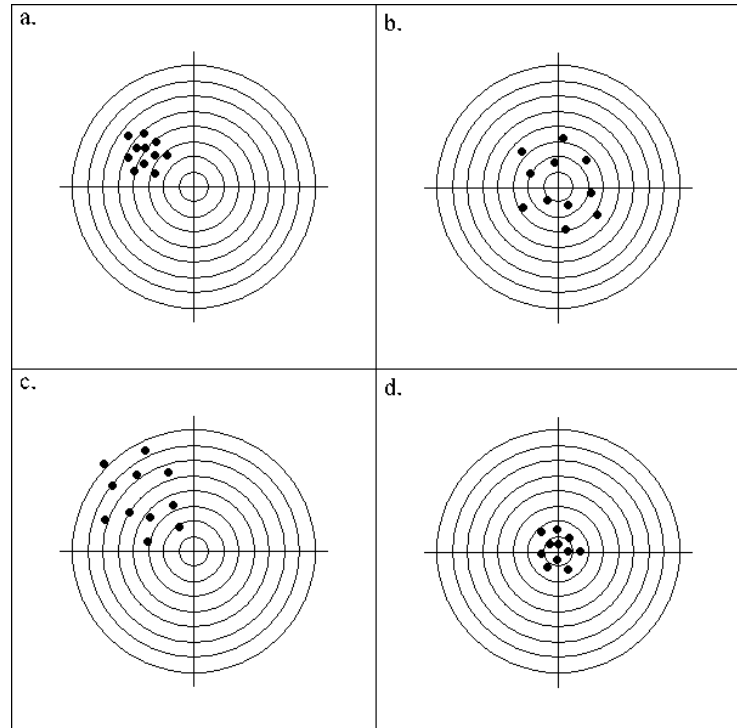

Fig. 1. (a) Estimated center of gravity is biased, but successive realizations (in the presence of noise) show a small dispersion. (b) Estimated center of gravity is unbiased, but highly dispersed. (c) Estimated center of gravity is biased, and highly dispersed. (d) Optimal situation, where the estimated center of gravity is unbiased and successive realizations show a small dispersion.

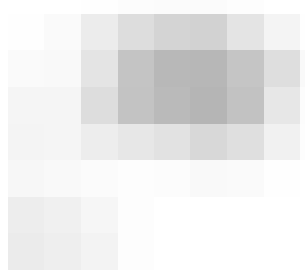

(a)

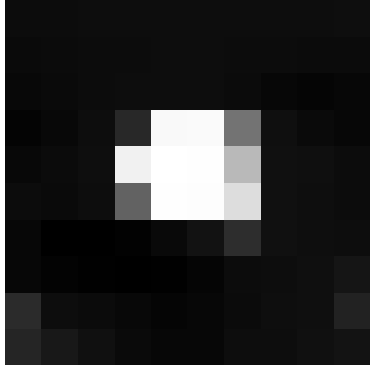

(b)

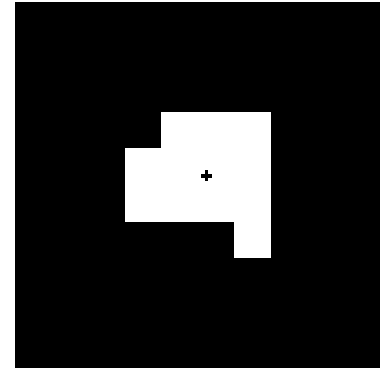

(c)

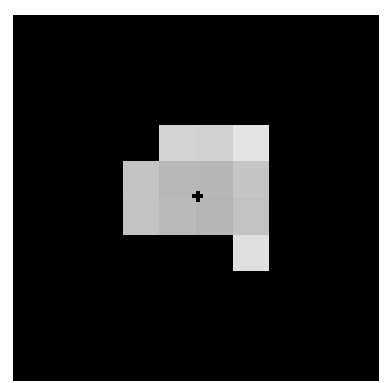

(d)

Fig. 2 (a) Output image resulting from a convolution with a kernel for detection of radiopaque markers. (b) shows the pixels of which the intensity exceeds the chosen threshold. (c) indicates the center of gravity (black cross) computed from the binary image (which results from a thresholding operation) using Eq. (2). (d) indicates the center of gravity (black cross) computed from the gray level image (after thresholding) using Eq. (3).

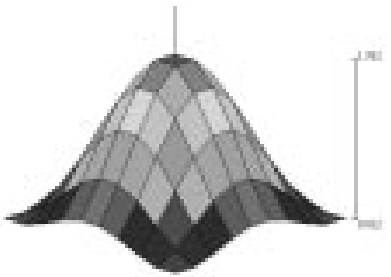

(a)

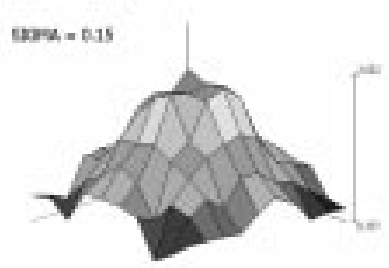

(b)

Fig. 3. (a) Artificial marker with a cosine profile. Gray levels range from 0 to 2. (b) the same marker superimposed with Gaussian noise with a standard deviation of 0.15 . 


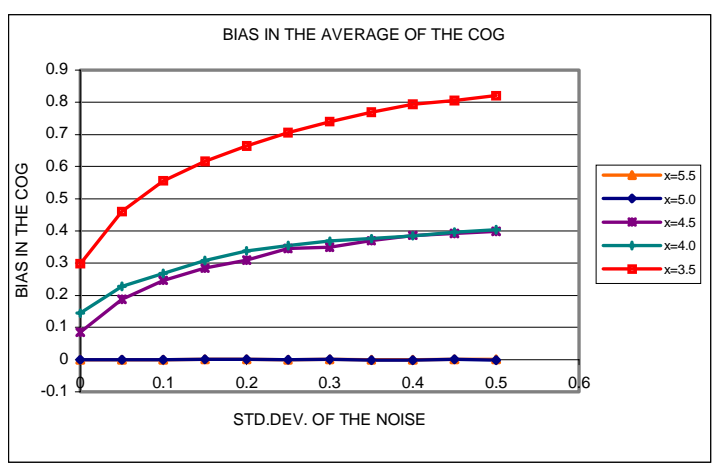

Fig. 4. Bias in the mean of the center of gravity (for the $x$-coordinate) of a marker cf. Fig. 3 as a function of the standard deviation of the noise in the gray levels. The different series indicate different window misplacements $(x=5.5$ means that the window was placed centrally on the marker). (a) without application of a threshold. (b) with application of a threshold.

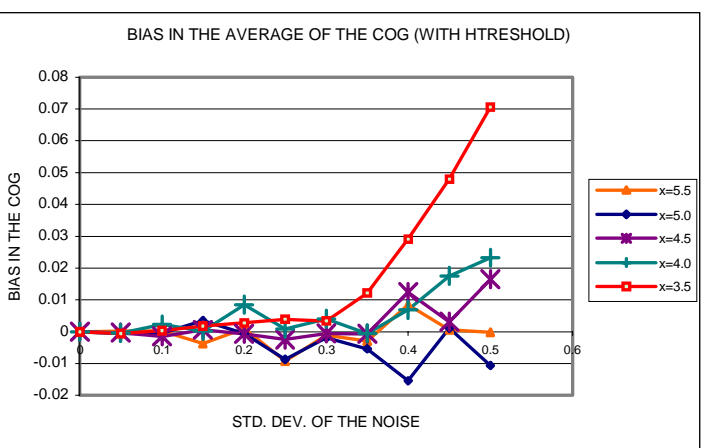

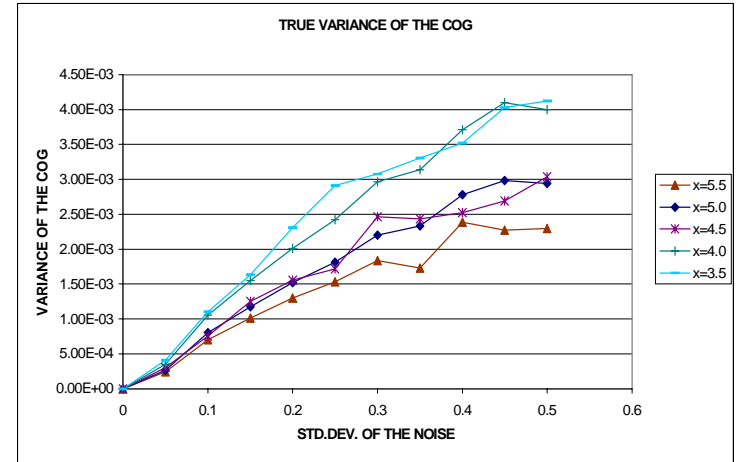

(a)

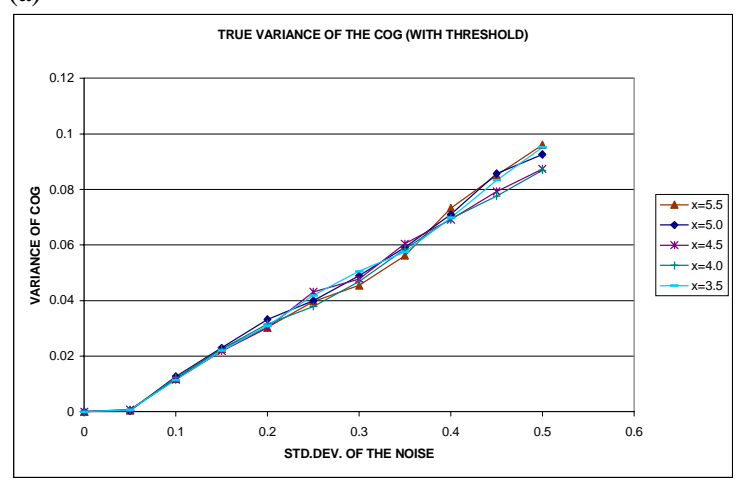

(c)

Fig. 5. Variances of the center of gravity of the marker cf. Fig. 3 as a function of the standard deviation of the noise in the gray levels. The different series indicate different window misplacements $(x=5.5$ means that the window was placed centrally on the marker). (a) true variance of the center of gravity from simulation data (no threshold). (b) errors of the estimated (cf. equation (11)) center of gravity with respect to the true variances (no threshold). (c) same as (a) but with threshold. (d) same as (b) but with threshold.

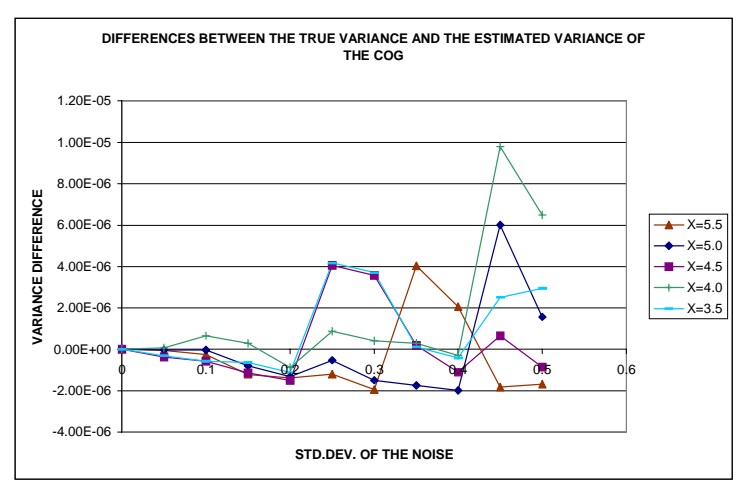

(b)

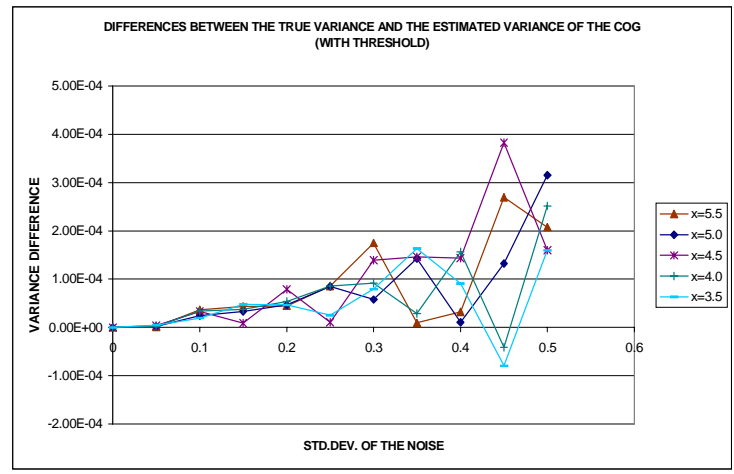

(d)

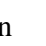
. 


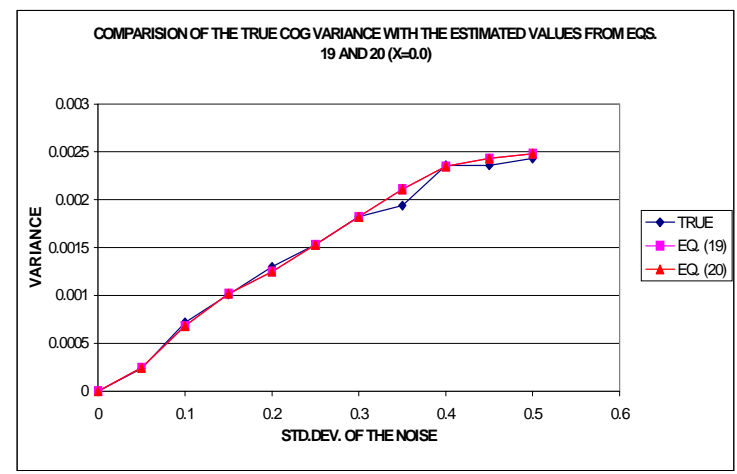

(a)

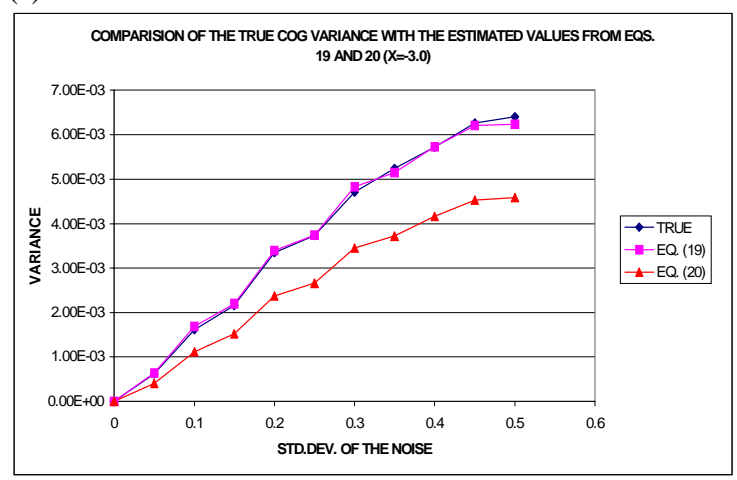

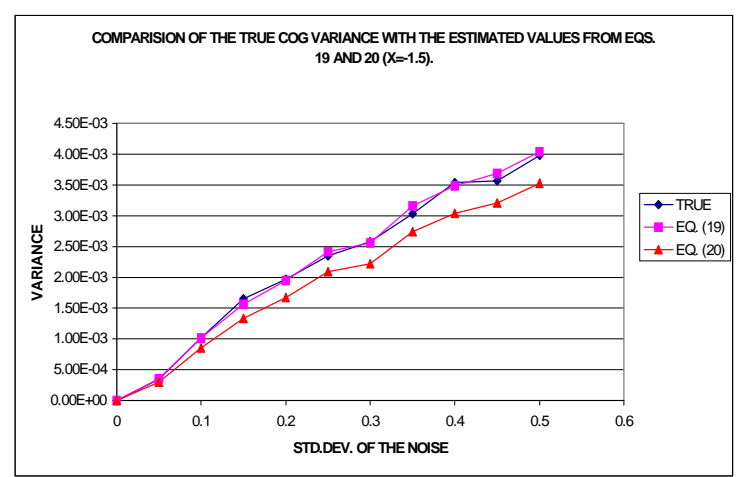

(b)

(c)

Fig. 6. Comparison of the true variance of the center of gravity from simulation data with values from equations (19) and (20). The origin of the coordinate system was placed at the center of gravity. (a) window was placed centrally on the marker. (b) window was misplaced along the $x$-direction by 1.5 pixels. (c) window was misplaced along the $x$-direction by 3.0 pixels.

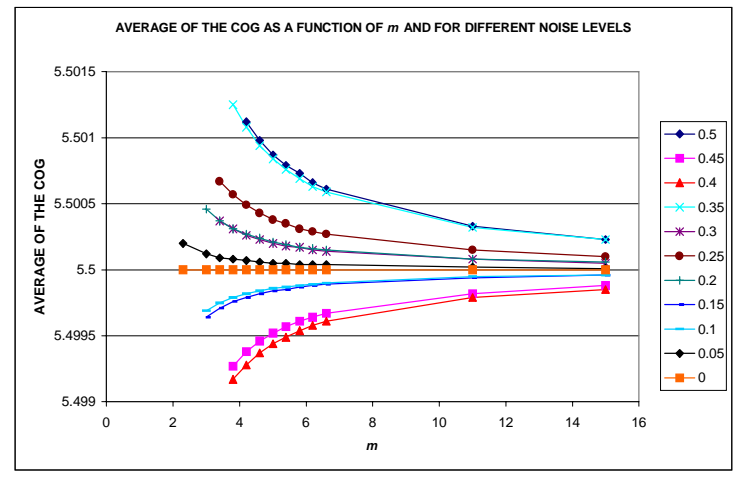

(a)

Fig. 7. The center of gravity and its variance as a function of the weighing parameter $m$ (equation (4)). The window was positioned centrally on the marker. Different series indicate different standard deviations of the noise in the gray values in the marker image. (a) center of gravity is more biased with larger difference between the maximum gray value and $m$. (b) the variance of the center of gravity (log-scale) decreases with increasing difference between the maximum gray value and $m$.

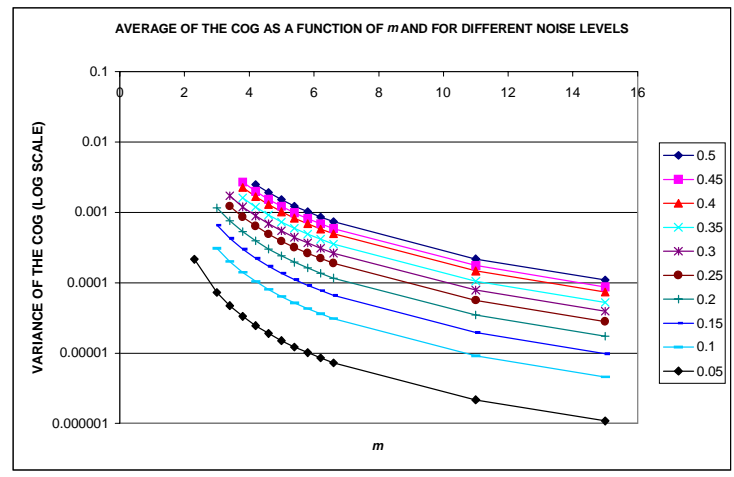

(b) 


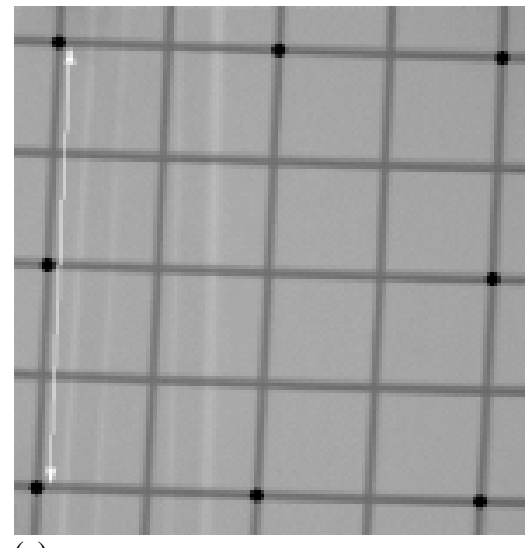

(a)

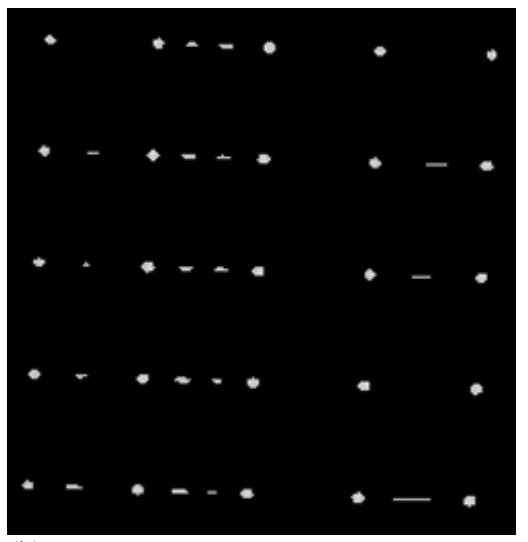

(b)

Fig. 8. (a) Radiograph of the rectangular grid used in experiment 6 . The band crossings used to measure the horizontal and vertical distances are indicated with black dots. All the distances measured were distances over 4 cells (white arrow illustrates the cord). (b) image indicating the detected grid crossings.

Table I. Results of two-sided Student's T- test with hypothesis $\mathrm{H}_{0}$ that two horizontal or vertical distances are equal. The critical value for $T$, with $1-\alpha=0.975$ and $v=18$, was 0.2101 . In the table, $d_{1}$ is the uppermost, $d_{2}$ the middle and $d_{3}$ the lowest horizontal distance and $\mathrm{d}_{4}$ is leftmost, $\mathrm{d}_{5}$ the middle and $\mathrm{d}_{6}$ the rightmost vertical distance. With decreasing SNR the hypothesis $\mathrm{H}_{0}$ can be rejected in a decreasing number of tests.

\begin{tabular}{|c|c|c|c|c|c|c|}
\hline \multicolumn{7}{|c|}{ Horizontal distances } \\
\hline & \multicolumn{2}{|c|}{$\mathrm{SNR}=24.3 \mathrm{~dB}$} & \multicolumn{2}{|c|}{$\mathrm{SNR}=18.3 \mathrm{~dB}$} & \multicolumn{2}{|c|}{$\mathrm{SNR}=12.3 \mathrm{~dB}$} \\
\hline $\mathrm{H}_{0}$ & T-value & Reject $\mathrm{H}_{0}$ ? & T-value & Reject $\mathrm{H}_{0}$ ? & T-value & Reject $\mathrm{H}_{0}$ ? \\
\hline$d_{1}=d_{2}$ & -3.53 & Yes & -1.82 & No & -1.03 & No \\
\hline$d_{2}=d_{3}$ & 3.83 & Yes & 2.17 & Yes & 1.24 & No \\
\hline$d_{1}=d_{3}$ & 0.51 & No & 0.47 & No & 0.29 & No \\
\hline \multicolumn{7}{|c|}{ Vertical distances } \\
\hline & \multicolumn{2}{|c|}{$\mathrm{SNR}=24.3 \mathrm{~dB}$} & \multicolumn{2}{|c|}{$\mathrm{SNR}=18.3 \mathrm{~dB}$} & \multicolumn{2}{|c|}{$\mathrm{SNR}=12.3 \mathrm{~dB}$} \\
\hline$\overline{\mathrm{H}_{0}}$ & T-value & Reject $\mathrm{H}_{0}$ ? & T-value & Reject $\mathrm{H}_{0}$ ? & T-value & Reject $\mathrm{H}_{0}$ ? \\
\hline $\mathrm{d}_{4}=\mathrm{d}_{5}$ & -3.59 & Yes & -2.21 & Yes & -0.94 & No \\
\hline$d_{5}=d_{6}$ & 0.43 & No & 0.27 & No & -0.51 & No \\
\hline $\mathrm{d}_{4}=\mathrm{d}_{6}$ & -3.02 & Yes & -1.85 & No & -1.31 & No \\
\hline
\end{tabular}

Table II. This table summarizes the results found in our simulation studies. The up-arrow in cell $(1,1)$, which combines bias with increasing window size, indicates a larger window will increase the bias in the center of gravity estimate compared with the true object center. The down-arrow in cell $(2,1)$, which combines variance with increasing window size, will decrease the variance in the estimated center of gravity. A 0 , e.g. in cell $(5,1)$, indicates that shifting the object has not effect on the bias when thresholding is applied before the center of gravity is computed.

\begin{tabular}{|l|l|l|l|l|l|}
\hline Factor & $\begin{array}{l}\text { Increasing } \\
\text { window size }\end{array}$ & Thresholding & $\begin{array}{l}\text { Poorer signal-to- } \\
\text { noise ratio }\end{array}$ & $\begin{array}{l}\text { Shifting object } \\
\text { (no threshold) }\end{array}$ & $\begin{array}{l}\text { Shifting object } \\
\text { (with threshold) }\end{array}$ \\
\hline Bias & $\uparrow$ & $\downarrow^{*} \uparrow^{* * *}$ & $\uparrow$ & $\uparrow$ & $\approx 0$ \\
\hline Variance & $\downarrow$ & $\uparrow$ & $\uparrow$ & $\uparrow$ & $\approx 0$ \\
\hline
\end{tabular}

